\title{
NY-ESO-1 Plasmid DNA Cancer Vaccine pPJV7611
}

National Cancer Institute

\section{Source}

National Cancer Institute. NY-ESO-1 Plasmid DNA Cancer Vaccine pPJV7611. NCI

Thesaurus. Code C62452.

A plasmid DNA encoding an immunogenic peptide derived from the cancer-testis antigen NY-ESO-1 with potential immunostimulating and antitumor activities. Upon administration, NY-ESO-1 plasmid DNA cancer vaccine pPJV7611 may stimulate the host immune system to mount a cytotoxic $T$ lymphocyte $(C T L)$ response against tumor cells expressing the NY-ESO-1 antigen, resulting in tumor cell lysis. NY-ESO-1 is a tumor associated antigen (TAA) found in normal testes and expressed on the surfaces of various tumor cells, including melanoma, breast, bladder, prostate, lung, ovarian, and hepatocellular tumor cells. 